\title{
LDV and PIV Measurements of the Organized Oscillations of Turbulent Flow over a Rectangular Cavity*
}

\author{
Takayuki MORI* $^{* *}$ and Kenji NAGANUMA ${ }^{* *}$ \\ ${ }^{* *}$ Naval Systems Research Center, TRDI/Ministry of Defense \\ 2-2-1, Nakameguro, Meguro-ku, Tokyo, JAPAN \\ E-mail: tmori@cs.trdi.mod.go.jp
}

\begin{abstract}
This paper presents results of an experimental study of the organized oscillation of incompressible turbulent flow over a rectangular cavity. Experiments were carried out to clarify the oscillation frequencies and the strength of shear layer-cavity trailing edge interaction. LDV and PIV measurements were made in a closed re-circulating water tunnel for rectangular cavities with $L / D$ ( $L$; cavity length, $D$; cavity depth) $=2$ and $L / D=4$. Measurements were also made for a backward facing step flow. Reynolds number based on momentum thickness was 8,300. LDV-measured velocity spectra reveal that self-sustained oscillations due to shear layer-trailing edge interaction are absent or very weak. At the upstream part of the cavity, dominant frequencies of the velocity fluctuations show little variation with $L / D$, and the frequencies are close to those of the shear layer instability. As the measuring point moves to the downstream, oscillation frequencies decrease and the peaks of the spectra become unclear. PIV-measured instantaneous flow fields show the existence of organized vortical structures in the cavity shear layer. From PIV images, frequencies of cavity oscillation are visually estimated and the results are compared to the LDV-measured velocity spectra.
\end{abstract}

Key Words: Turbulent Flow, Cavity Oscillation

\section{Introduction}

Flow over a cavity is known to induce undesirable noise and vibration in air ${ }^{(1)}$ and water ${ }^{(2)}$ borne vehicles. The flow field is extremely complex due to simultaneous occurrence and interactions of multiple fluid dynamic phenomena; shear layer development, flow impingement, unsteady re-circulation within the cavity. Therefore, the natures of flow oscillations strongly depend on many hydrodynamic and geometrical parameters, for example, Reynolds number, $\theta / D(\theta$; momentum thickness, $D$; cavity depth) and $L / D(L$; cavity length). At present, it is safe to say that our knowledge is not sufficient to predict oscillation frequencies of cavity flows in practical applications. In particular, results for turbulent cavity flows at high Reynolds number, $R e_{\theta}>5000$, are scarce.

The primary purpose of this study is to extend the understanding of unsteady characteristics of turbulent cavity flows at high Reynolds number, particularly on their oscillation frequencies and strength of shear layer-cavity trailing edge interaction. LDV and PIV measurements were made for rectangular cavities with $L / D=2$ and $L / D=4$ at $R e_{\theta}=8,300$ and $\theta / D=0.04$. To assess the strength of shear layer- trailing edge interaction, measurements were also made for a backward facing step (BFS) flow. The results reported in this paper

Received 18 Feb., 2010 (No. 10-0094)

Copyright $(C 2010$ by JSME 
will provide a comprehensive data set on unsteady characteristics of turbulent cavity flows when combined with the results on three-dimensional characteristics in a previous paper ${ }^{(3)}$.

Although most of cavity flows in engineering applications are turbulent, relatively small number of investigations ${ }^{(4)-(6)}$ has been done for incompressible cavity flows with turbulent boundary layer upstream. A detailed PIV measurement on turbulent cavity flow has been made by Lin and Rockwell ${ }^{(4)}$ at $R e_{\theta}=1370, \theta / D=0.05$. They have pointed out that two instabilities, small- and large-scale instability, exist in the turbulent cavity flow. From time series analysis of pressure fluctuations, they found that the frequency of the large-scale instability was coincident with the most unstable frequency of the separated shear layer. Chang et.al, ${ }^{(5)}$ have performed a LES (Large Eddy Simulation) analysis on incompressible cavity flow with a thick turbulent boundary layer $\left(\theta / D=0.275, \operatorname{Re}_{\theta}=920\right)$ upstream. When frequencies are normalized by the momentum thickness, peak frequencies in their paper are higher than that predicted from linear stability theory. In their case, it appears that cavity depth and length are more adequate to describe the oscillation frequencies. More recently, Kang and Sung ${ }^{(6)}$ have performed wind tunnel experiments on low Mach number turbulent cavity flows for various $L / D, \theta / D$ and Reynolds numbers up to $R e_{\theta}=2900$. They demonstrated that the natures of the cavity oscillations severely varied with these parameters.

The reminder part of this paper consists of four sections. Section 2 describes experimental setup and technique. Section 3 presents experimental results and discussion. Following a brief review on turbulence statistics, LDV-measured oscillation frequencies are discussed. The details of instantaneous flow fields are presented in the last part of the section. The final section summarizes the results.

\section{Nomenclature}

$D \quad$ : cavity depth

$E_{X}(f)$ : power spectral density of $X$

$f \quad:$ frequency

$L \quad$ : cavity length

$q$ : dynamic pressure of the free stream

$R e_{\theta}: \quad$ Reynolds number based on momentum thickness

$S t_{D}$ : Strouhal number based on cavity depth $\left(=f D / U_{0}\right)$

$S t_{\theta}$ : Strouhal number based on momentum thickness $\left(=f \theta / U_{0}\right)$

$U_{0}$ : free stream velocity at the cavity inlet

$U_{c}:$ convective velocity

$u$ : streamwise velocity

$v$ : vertical velocity

$x: \quad$ streamwise coordinate

$y$ : vertical coordinate

$z$ : spanwise coorinate

$\delta_{\omega}: \quad$ vorticity thickness $\left(=U_{0} /(\partial u / \partial y)_{\max }\right)$

$\lambda_{x}:$ streamwise wave length

$\theta:$ momentum thickness of the boundary layer 


\section{Experimental apparatus and technique}

Experiments were carried out in a closed re-circulating water tunnel at the Naval Systems Research Center, TRDI/Ministry of Defense, Japan. The test section of the tunnel is 233 $\mathrm{mm}(\mathrm{W}) \times 233 \mathrm{~mm}(\mathrm{H}) \times 1160 \mathrm{~mm}(\mathrm{~L})$. Figure 1 shows experimental setup ${ }^{(3)}$. By varying the location of the downstream liner, cavity length was varied. In this paper, the origin of the coordinates is defined as the mid-span of the cavity leading edge. All velocity measurements in this paper were carried out at the cavity mid-span.

Present experiments were carried out in three phases. In the first phase, LDV measurements were carried out, and the water of the tunnel was seeded with titanium oxide particles of $1 \mu \mathrm{m}$ diameter. A two-component fiber-optic LDV system was used with forward-scatter configuration. The LDV system consisted of a fiber optic probe (TSI Model 9832), a corner cube (TSI Model 9240) and a digital Spectrum analyzer (TSI IFA750). Control volume of the probe was approximately $1.6 \mathrm{~mm}$ length and $0.11 \mathrm{~mm}$ diameter. Blue $(\lambda=488 \mathrm{~nm})$ and green $(\lambda=514 \mathrm{~nm})$ Argon-ion laser beams were used for the streamwise and vertical velocity measurements, respectively. To keep sufficient data rate for spectral analysis, the LDV system was operated with non-coincident mode. The data rate of the LDV measurements varied between $500 \mathrm{~Hz}$ and $3000 \mathrm{~Hz}$. Velocity spectra were calculated from auto-correlation functions of the velocity fluctuations because LDV measurements are not continuous. Velocity spectra of this paper were calculated from 10 individual FFT's.

In the second and third phase, $x-y$ and $x-z$ plane PIV measurements were carried out. For PIV measurements, tunnel water was seeded with polyamide particles of $10 \mu \mathrm{m}$ diameter. The PIV system consisted of a double pulse Nd-Yag laser (TSI Mini-Twin $\lambda=532 \mathrm{~nm}, 30$ $\mathrm{mJ} /$ pulse), and a CCD camera (TSI Model-630046, $1280 \times 1024$ pixels, 12bit resolution). Post processing of the captured images was carried out by commercial software (LaVision, Davis7.0). Particle displacements were calculated with a multi-pass algorithm and the final window size was $32 \times 32$ pixels with $50 \%$ overlap. Validation of calculated vectors was done based on correlation ratio between the first and the second peak. Vectors with low correlation ratio less than 1.3 were removed. Typical displacement of the particle was 5 pixels. Turbulence statistics of the cavity flows were calculated from 600 PIV images. The

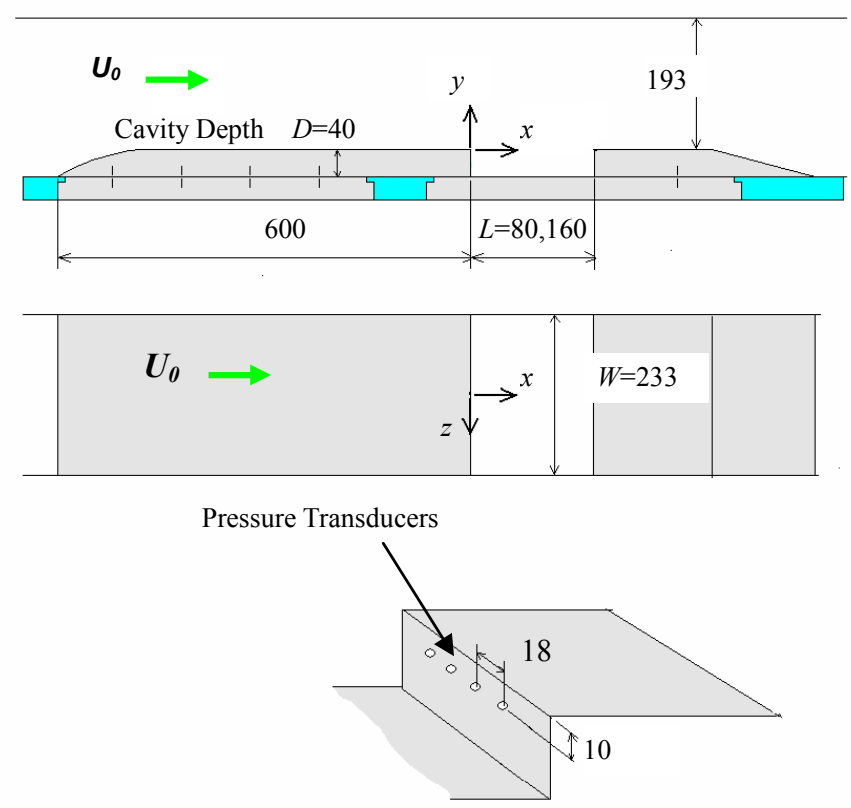

Figure 1 Experimental setup and coordinates. (unit: mm) 
uncertainty in the PIV measurement was estimated by considering a characteristic sub-pixel resolution of \pm 0.3 pixels and standard deviation of repeated measurements. Based on $95 \%$ confidence interval, uncertainty in the time averaged velocity, turbulence intensity and Reynolds stress were estimated to be $\pm 2 \%, \pm 6 \%$ and $\pm 7 \%$, respectively.

Figure 2 shows direct comparisons LDV and PIV measurements on streamwise turbulence intensity and normalized Reynolds stress. The results were measured for the BFS flow at $x / D=0.8$, and image magnification was 400 pixels $/ D$ (cavity depth). Although each result shows similar trend, LDV-measured turbulence intensity is $30 \%$ higher than that measured by PIV at $y / D \sim 0$. This trend is consistent with a prior measurement ${ }^{(8)}$ on an inclined BFS flow. It is likely that the larger PIV measuring volumes suppress the values of $u_{r m s}^{\prime}$. This discrepancy in $u_{r m s}^{\prime}$ decreased at the downstream locations. At $x / D=1.6$, the largest discrepancy was estimated to be $10 \%$ of the maximum value of $u^{\prime}{ }_{r m s}$.

In addition to the velocity measurements, pressure fluctuations at the cavity rear wall were measured in the second and third phase of the experiments. Piezoelectric pressure transducers of 5.5mm (PCB HSM112A22) were flush mounted to the cavity rear wall. All results of this paper were obtained with a pressure transducer located at the cavity mid-span. The vertical location of the transducer was $0.25 \mathrm{D}$ below the cavity trailing edge. Signals from the transducer were acquired using an A/D converter (Ono-Sokki, DS2000) at 12.5 $\mathrm{kHz}$ sampling rate.

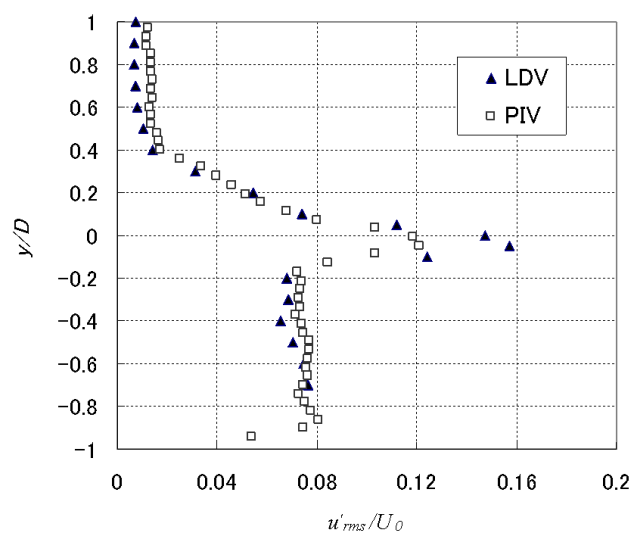

(a) Streamwise mean velocity fluctuation.

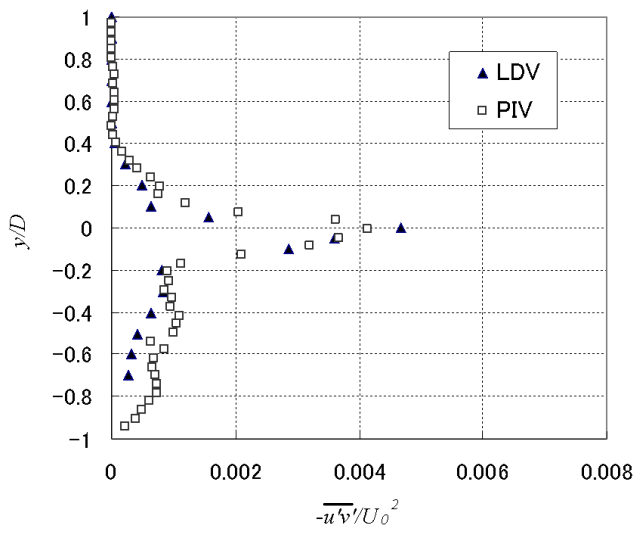

(b) Normalized Reynolds stress.

Figure 2 Comparison of LDV and PIV measured turbulence statistics for the backward facing step flow. $\left(U_{0}=5.0 \mathrm{~m} / \mathrm{s}, x / D=0.8\right)$

\section{Results and discussion}

\subsection{Turbulence Statistics}

Figure 3 shows LDV-measured velocity profiles at the inlet of the cavities and the backward facing step (BFS). The velocity profiles at the upstream edge are essentially identical between the three geometrical configurations. The momentum thickness $\theta$ of the boundary layer was determined to be $1.6 \mathrm{~mm}^{(3)}$, and Reynolds number based on momentum thickness was $8,300 \pm 300$ considering the variation of water temperature.

Turbulence statistics derived from PIV measurements are presented in figures 4-6. At the bottom walls of the cavities, strong reverse wall-jet can be observed. Regions with high turbulence intensity levels can be found at the inside of the cavity, in addition to the inside of the cavity shear layer. Shear layer of the longer cavity develops more rapidly than that of the shorter cavity. It is possibly that re-circulating flow within the cavity has strong influences on the shear layer development. 


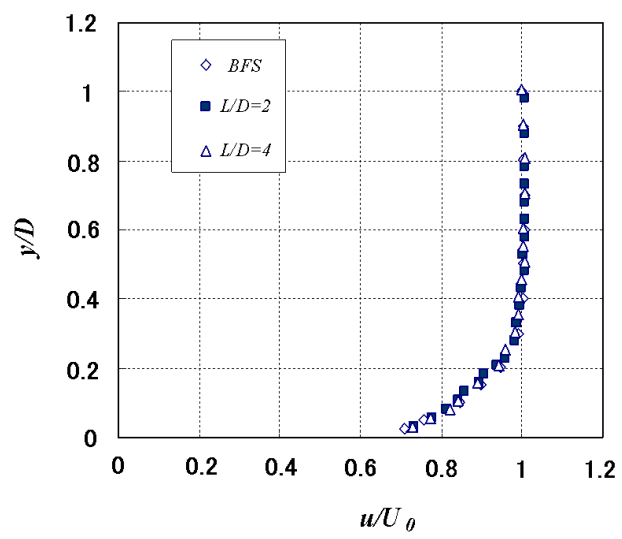

Figure 3 Velocity profiles of the boundary layers at the cavity inlet. $\left(U_{0}=5.0 \mathrm{~m} / \mathrm{s}\right)$

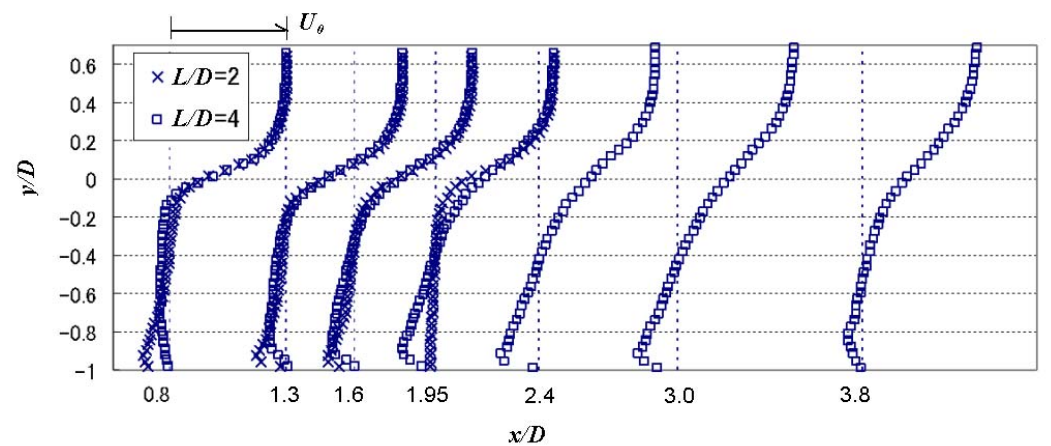

Figure 4 PIV-measured mean streamwise velocity profiles.

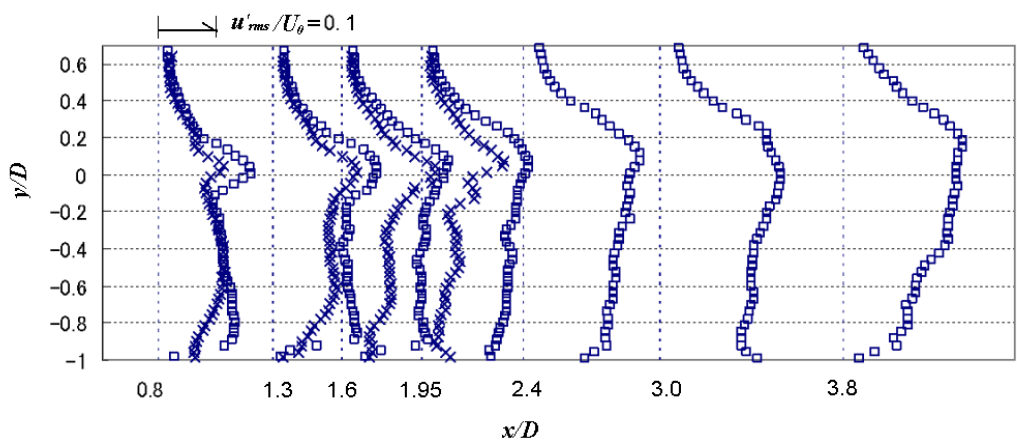

(a) Streamwise component

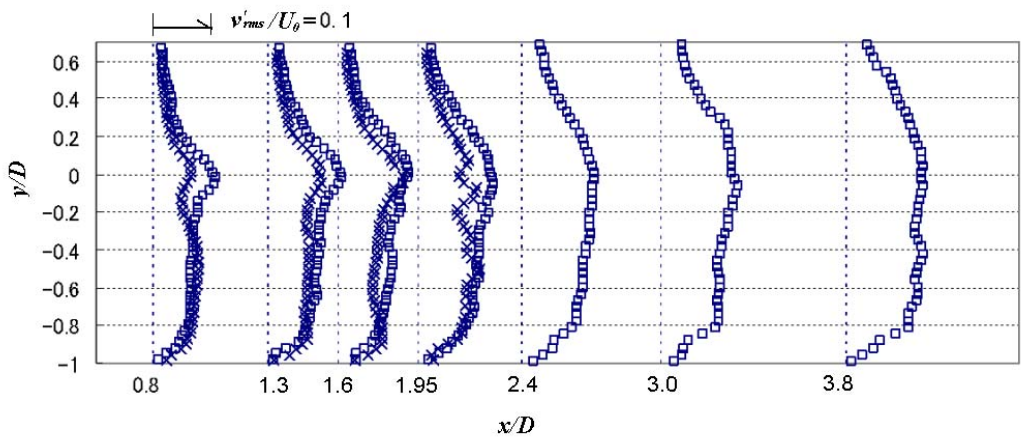

(b) Vertical component

Figure 5 PIV-measured turbulence intensities. The symbols are the same as figure 4 . 


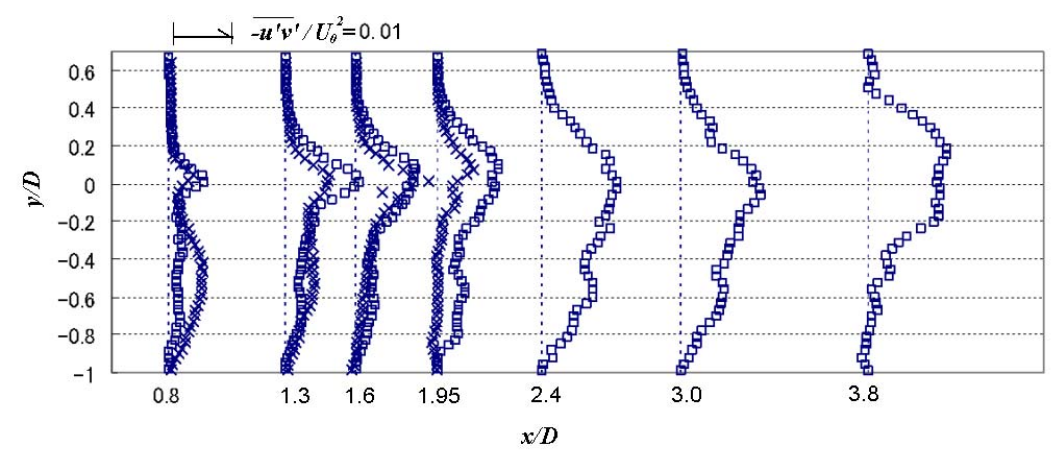

Figure 6 PIV-measured normalized Reynolds shear stress. The symbols are the same as figure 4 .

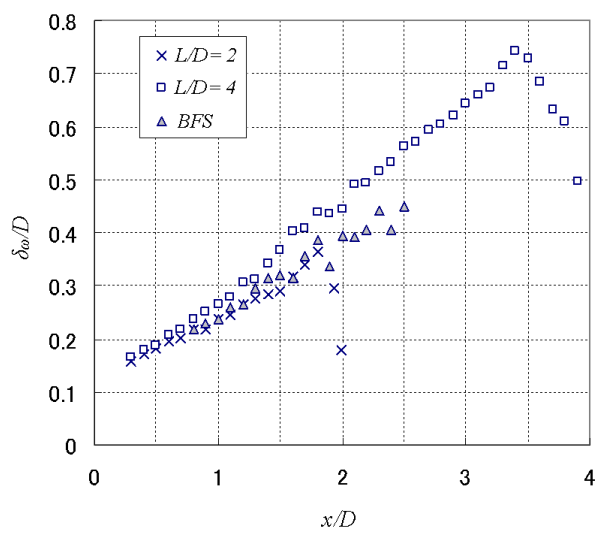

Figure 7 Variation of vorticity thickness $\delta_{\omega}$ with $x / D$.

In the present study, shear layer spreading rate was evaluated from vorticity thickness $\delta_{\omega}$. Vorticity thickness $\delta_{\omega}$ is defined as $\delta_{\omega}=U_{0} /(\partial u / \partial y)_{\max }$. Figure 7 shows variations of $\delta_{\omega}$ with $x / D$. In the figure, results for the backward facing step flow are included for comparison. In the figure, results for $x / D<0.3$ are omitted because relatively large discrepancies of $10 \%$ are found between the PIV and LDV results. It can be seen that the shear layers spread linearly, as reported in prior studies for both laminar ${ }^{(7)}$ and turbulent ${ }^{(5)}$ cavity flows. Shear layer spreading rate $\mathrm{d} \delta_{\omega} / \mathrm{dx}$ is 0.18 for $L / D=4$ and 0.14 for $L / D=2$. As expected from turbulence statistics, shear layer spreads more rapidly for the longer cavity. Shear layer spreading rate of the longer cavity is higher than that of the backward facing step flow.

Prior studies ${ }^{(5)(9)}$ have reported that shear layer spreading rate of cavity flows are higher than those of free mixing layers. For example, Chang et.al ${ }^{(4)}$ reported that $\mathrm{d} \delta_{\omega} / \mathrm{dx}$ of a incompressible turbulent shear layer was 0.35 while a typical spreading rate for turbulent mixing layer is $0.162^{(10)}$. However, measured shear layer spreading rates in the present experiment are close to those of turbulent mixing layers. Although shear layer spreading rates slightly increase at $x / D>1, \mathrm{~d} \delta_{\omega} / \mathrm{dx}$ in the present experiment are lower than 0.2 . In addition, shear layer spreading rate for the shorter cavity is approximately the same as that of the backward facing step flow. These results indicate that the interaction between shear layer and cavity trailing edge is not strong enough to enhance the development of the cavity shear layer.

\subsection{Oscillation frequencies}

Figure 8 shows normalized vertical velocity spectra measured at the upstream part of the cavities. Results measured for the backward facing step flow are included for comparison. In the figure, frequency $f$ and power spectral density $E_{u}(f)$ are normalized by the free stream 


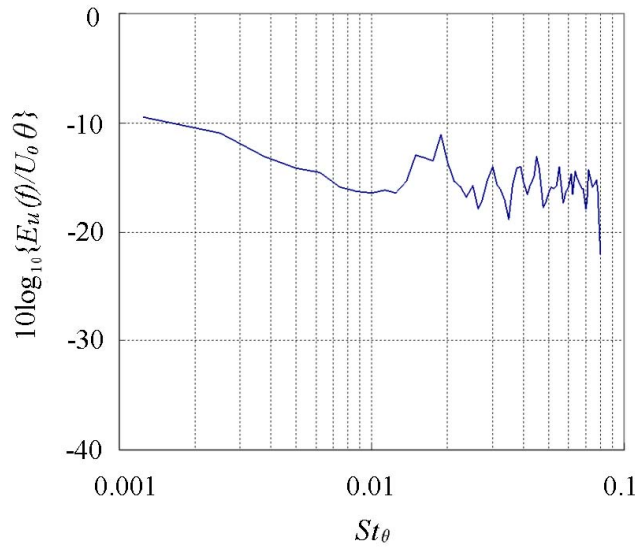

(a) $L / D=2, x / D=1.0, y / D=0.125$

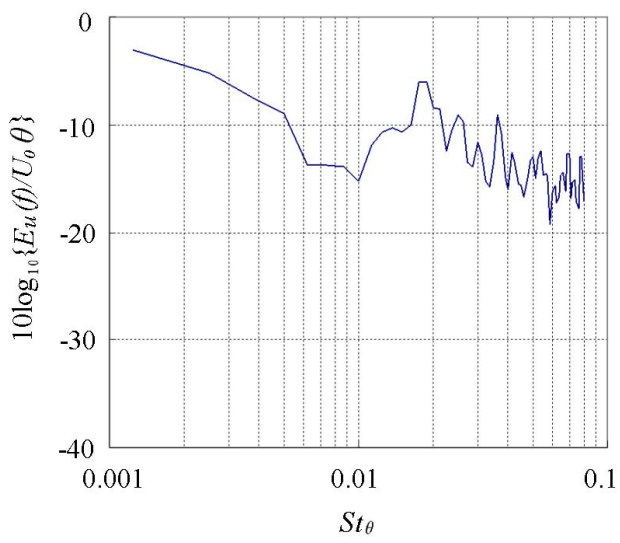

(b) $L / D=4, x / D=0.8, y / D=0.1$

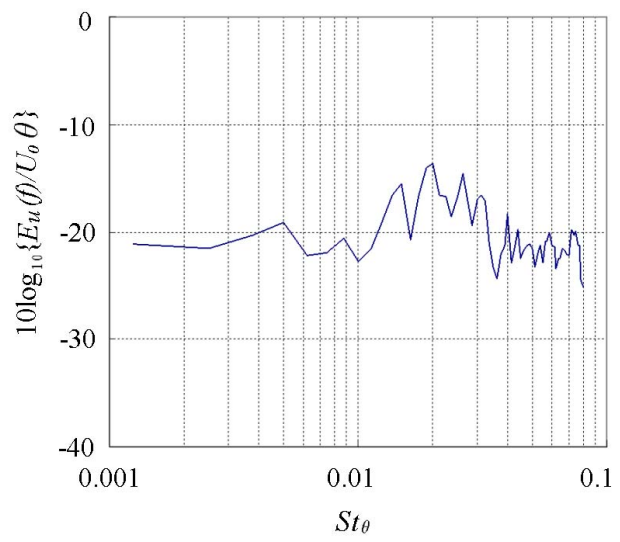

(c) BFS, $x / D=1.2, y / D=0.125$

Figure $8 \mathrm{LDV}$-measured power spectra of vertical velocity fluctuations.

These data were taken at the upstream part of the separated shear layer.

velocity $U_{0}$ and the momentum thickness $\theta$. The velocity spectra were obtained from auto-correlation functions of LDV signals using FFT (fast Fourier transform) algorithm. The vertical location for each measurement was determined by considering the data rate required for the spectral analysis. Because LDV data rates are approximately proportional to the velocity, locations at the high-speed side (near the free-stream flow) of the shear layer 
were chosen for the measurements.

Each spectrum in fig. 8 shows that the dominant frequency corresponding to the largest peak lies at $S t_{\theta}\left(=f \theta / U_{0}\right) \sim 0.018$. The non-dimensionalized frequency $S t_{\theta} \sim 0.018$ is close to the most unstable frequency based on inviscid stability theory. Previous studies on laminar $^{(7)}$ and turbulent ${ }^{(4)}$ cavity flows have reported the occurrences of dominant peaks at the similar range of $S t_{\theta}$. In addition to the largest peaks, additional peaks are also

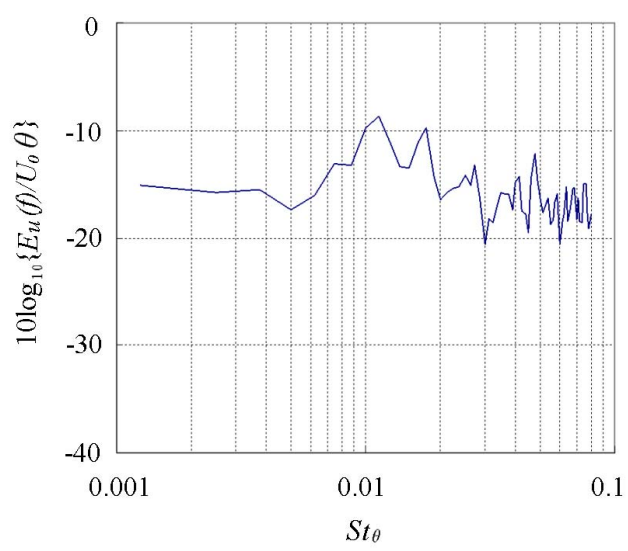

(a) $L / D=2, x / D=2.0, y / D=0.25$

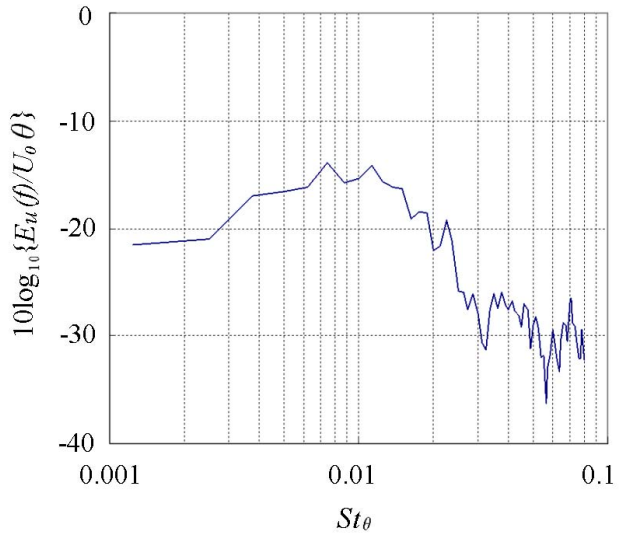

(b) $L / D=4, x / D=3.6, y / D=0.6$

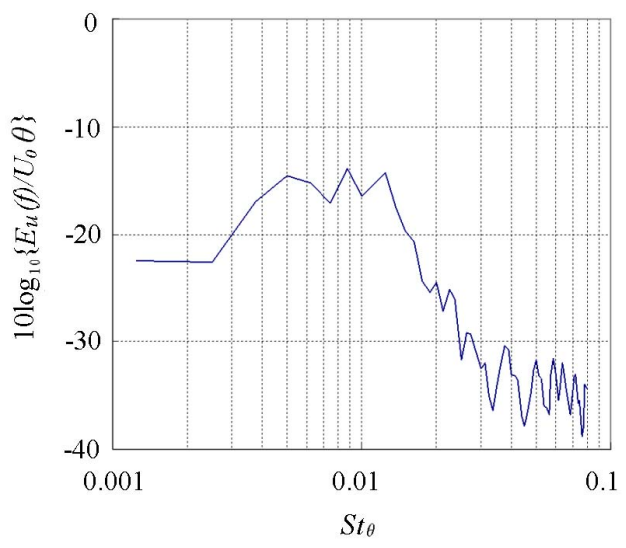

(c) BFS, $x / D=4.8, y / D=0.125$

Figure $9 \mathrm{LDV}$-measured power spectra of vertical velocity fluctuations. These data were taken at the downstream part of the separated shear layer. 


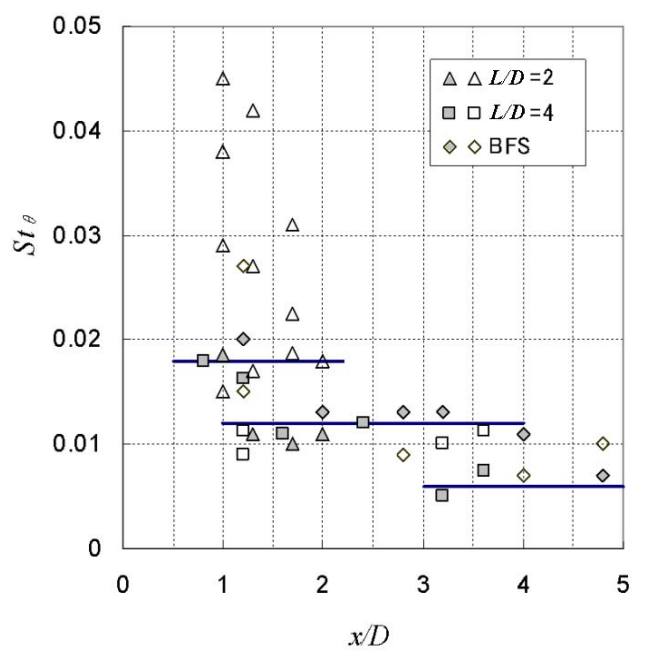

Figure 10 Peak frequencies of the velocity spectra as a function of $x / D$. The filled marks indicate dominant frequencies corresponding to the largest peaks in the velocity spectra.

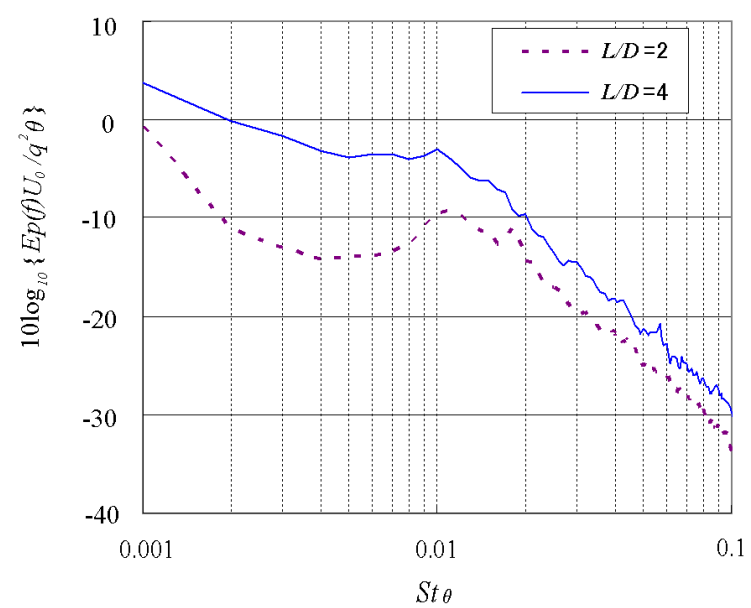

Figure 11 Normalized pressure spectra measured at the rear wall of the cavities.

identifiable at higher frequencies. In the present experiment, peak frequencies of the spectra were time-varying. In the individual FFT's before averaging, dimensionless frequency $S t_{\theta}$ of the largest peak varied between 0.016 and 0.02 . It is probably that the unsteadiness of the inlet boundary layer results in the frequency modulation of the velocity fluctuation. It should be noted that the dominant frequency found in the backward facing step flow is approximately the same as those found in the cavity flows. This observation suggests that the dominant frequency at the upstream part of the separated shear layer is essentially governed by the nature of the upstream boundary layers. Comparing the strength of the peaks at the dominant frequencies, there are no significant differences between the results of the cavity flows and that of the backward facing step flow. This observation indicates that the presence of cavity trailing edge does not enhance the organization of the vortical structures in the separated shear layer, in contrast to a finding reported for laminar inflow ${ }^{(7)}$.

Figure 9 shows normalized vertical velocity spectra measured at the downstream locations. In the case of $L / D=2$, the dominant frequency decreases from $S t_{\theta} \sim 0.018$ to $S t_{\theta}$ 
$\sim 0.011$ as the measuring location moves from $x / D=1$ to $x / D=2$. A sub-dominant peak is identified at $S t_{\theta}=0.018$ in the spectra measured at $x / D=2$. In the case of $L / D=4$ and BFS, velocity spectra at the downstream locations show different features. In the spectra, dominant peaks are unclear, and fluctuating energy significantly increase in a low-frequency range of $0.003<S t_{\theta}<0.01$.

Figure 10 shows peak frequencies of the velocity spectra as a function of $x / D$. In addition to the largest peaks of the spectra, peaks identified within $3 \mathrm{~dB}$ of the maximum spectral power are included. It is evident that the peak frequencies at the downstream locations are lower than those found at the upstream locations. At the upstream locations, $x / D<1.2$, the largest amplitude peaks are found at $S t_{\theta}=0.018 \pm 0.002$. Then, the dominant frequencies decrease to $0.012 \pm 0.002$ at $1.2<x / D<4$ and $0.006 \pm 0.002$ at $3.2<x / D<4.8$. When the frequencies are normalized by cavity depth $D$, the dominant frequencies at $1.2<x / D<4$ are non-dimensionalized as $S t_{D}=0.3 \pm 0.05$. The values of $S t_{D}$ are little lower than that found in LES analysis (Chang et.al. ${ }^{(5)}, S t_{D}=0.38$ ) and little higher than those found in wind tunnel experiments (Kang and Sung ${ }^{(6)}, S t_{D}=0.25 \sim 0.275$, the values varied with $\theta / D$ ).

Figure 11 shows pressure spectra measured at the rear wall of the cavities. In the figure, pressure spectra are normalized by $U_{0}, \theta$, and free stream dynamic pressure $q$. Although measured pressure spectra are essentially broadband, several peaks can be found. In the case of $L / D=2$, the primary and secondary peaks are identified at $S t_{\theta}=0.011$ and $S t_{\theta}=0.018$ respectively. For the longer cavity, a weak peak can be identified at $S t_{\theta}=0.01$. These peak frequencies in the pressure spectra are nearly coincident with those found in the velocity spectra measured at the downstream part of the cavities.

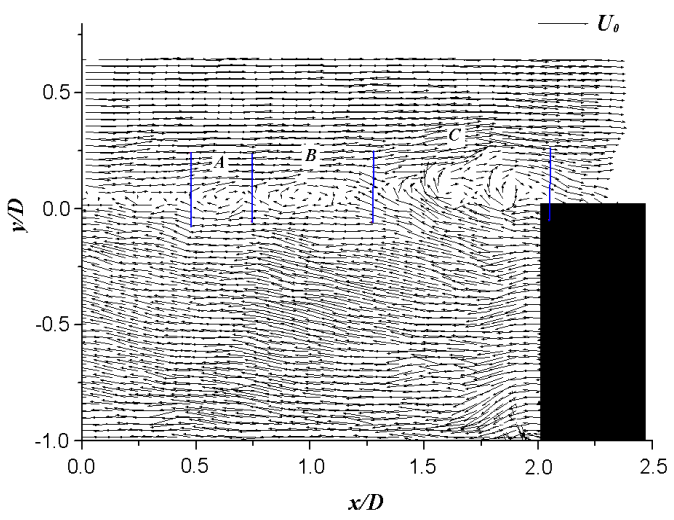

(a) Vector field

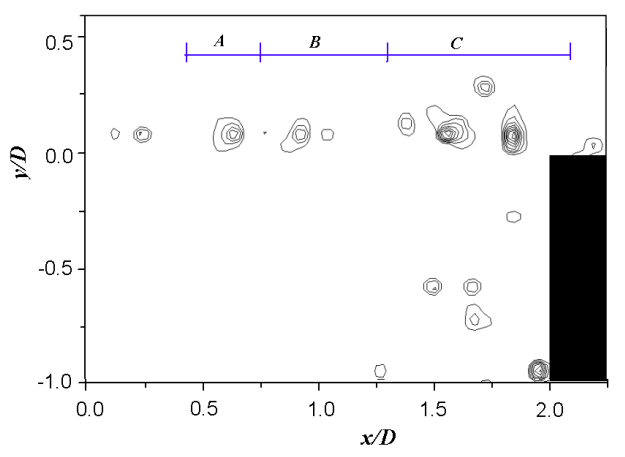

(b)Swirling strength

Figure 12 Instantaneous vector field and corresponding contours of the swirling strength for the cavity of $L / D=2$. The vectors are shown in a reference frame moving at $0.4 U_{0}$. Interval of the contour is $4.8(1 / \mathrm{s})$. 


\subsection{Instantaneous Flow Fields}

Figures 12 and 13 show representative examples of instantaneous flow fields, and corresponding contours of the swirling strength. The vectors are shown in a reference frame moving at $0.4 U_{0}$. In the present study, swirling strength is used to identify vortical structures in the cavity shear layer. The swirling strength is the imaginary portion of the eigenvalue of the local velocity gradient tensor. It is commonly accepted that the swirling strength is useful to identify vortical structures in highly shear flows ${ }^{(11)}$.

Using the reference frame, organized swirling structures can be identified in the vector field, as labeled A, B and C. The flow patterns in the moving frame are similar to a prior result ${ }^{(4)}$ obtained at a lower Reynolds number. As the organized structures move to the downstream, they grow into larger structures. As expected from the result of the vorticity thickness, vertical widths of the structures of the longer cavity are larger than those of the shorter cavity.

In the contours of the swirling strength, smaller structures can be identified in the cavity shear layer. The small-scale structures are formed from the cavity leading edge as reported by Lin and Rockwell ${ }^{(4)}$. From fig.12, it can be seen that the large-scale structure labeled C consists of multiple small-scale structures. At the upstream part of the cavity, interaction and merging of the small-scale structures were observed in the PIV images. It is likely that the small-scale structures in the cavity shear layer are responsible for the occurrences of high-frequency peaks in the velocity spectra. It should be addressed that these vortical

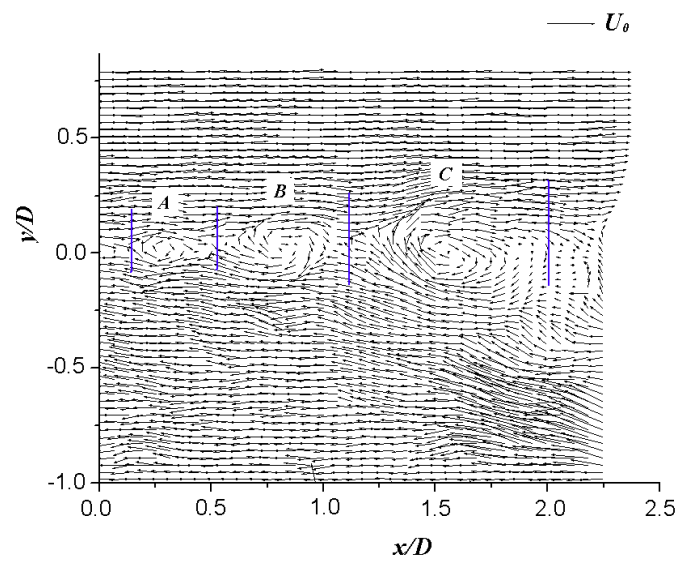

(a) Vector field

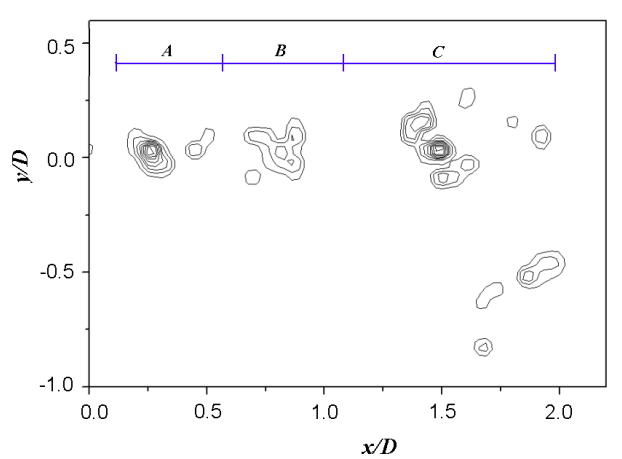

(b)Swirling strength

Figure 13 Instantaneous vector field and corresponding contours of the swirling strength for the cavity of $L / D=4$. The vectors are shown in a reference frame moving at $0.4 U_{0}$. Interval of the contour is $4.8(1 / \mathrm{s})$. 
structures are strongly distorted in the spanwise direction. As reported previously ${ }^{(3)}$, high and low speed streaks were observed in the cavity shear layer. In the case of the cavity with $L / D=2$, mean spanwise spacing of the high speed streaks was $0.35 D\left(\sim \delta_{\omega}\right)$.

From mean streamwise wavelength $\lambda_{x}$ and covective velocity $U_{c}$ of the large-scale structures, oscillation frequencies were visually estimated. In the present study, $U_{c}$ was determined by averaging streamwise velocity at the center of the large-scale structures. In the case of $L / D=2, \lambda_{x}$ and $U_{c}$ were estimated to be $0.8 D$ and $0.4 U_{0}$ at $1<x / D<2$. Using the $\lambda_{x}$ and $U_{c}$, oscillation frequency at $x / D=1.5$ was estimated to be $S t_{\theta}=0.02$. The visually estimated frequency is nearly coincident with the dominant frequency of the velocity fluctuations at $x / D<1.2$. Although velocity spectra measured at $1<x / D<2$ showed lowfrequency peaks at $S t_{\theta}=0.012 \pm 0.002$, instantaneous flow fields did not show large streamwise wavelengths corresponding to the low-frequency components. In the case of laminar impinging shear layer ${ }^{(12)}$, Knisely and Rockwell have observed low-frequency fluctuations associated with a cycling between different patterns of vortex-edge interaction. Further investigations, for example time-resolved PIV measurements, are required to clarify the detail of the low-frequency components of the cavity oscillations.

Fig.14 shows an example of instantaneous flow field at the downstream part of the longer cavity. At the downstream part of the separated shear layer, velocity spectra exhibit weak peaks at low-frequencies as shown in fig.9. In the vector field, clockwise rotating structures are observed in the cavity shear layer. From the contours of the swirling strength, it can be seen that small vortical structures distribute randomly. It appears that vortex structures at

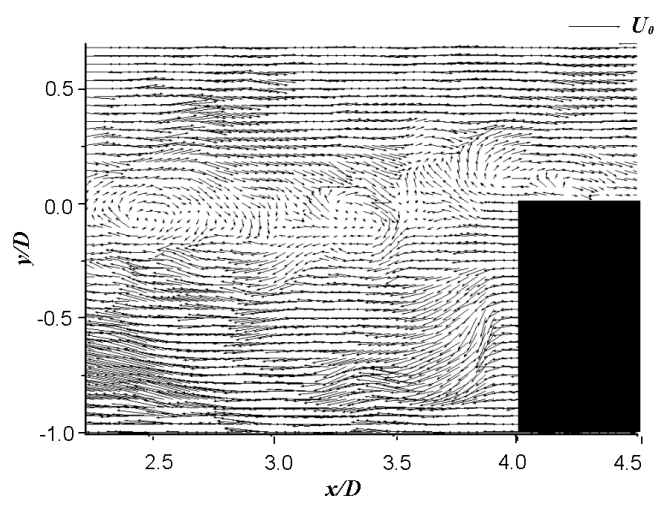

(a) Vector field

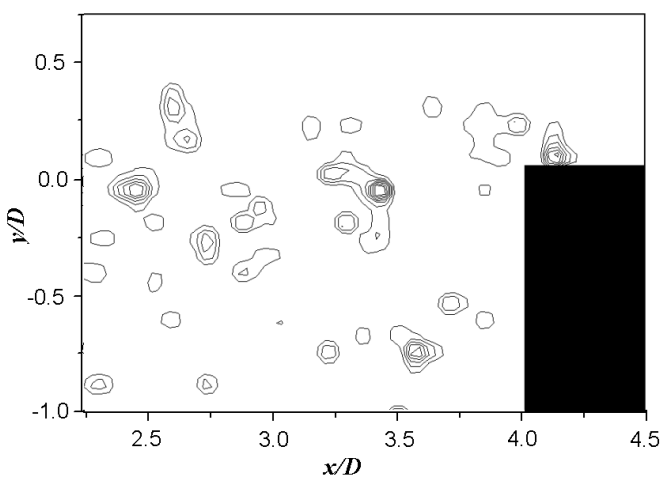

(b) Swirling strength

Figure 14 Instantaneous vector field and corresponding contours of the swirling strength measured at the downstream part of for the cavity of $L / D=4$. The vectors are shown in a reference frame moving at $0.4 U_{0}$. Interval of the contour is $2.2(1 / \mathrm{s})$. 
the downstream locations are less organized than those at the upstream locations. It is possible that the lack of the organization of the cavity shear layer result in the occurrences of the low-frequency components of the velocity fluctuations.

\section{Conclusions}

An experimental study on turbulent cavity flow was carried out to clarify the oscillation frequencies and the strength of shear layer-cavity interaction. Main results are summarized as follows.

(1) Shear layer spreading rates derived from velocity profiles indicate that shear layer-cavity trailing edge interaction does not enhance the shear layer development. Shear layer spreading rates of the cavity flows are approximately the same as that of the backward facing step flow, and the values are close to a typical value of free mixing layers. Results on turbulence statistics suggest that re-circulating flow within the cavity has strong influences on the development of cavity shear layer.

(2) LDV-measured velocity spectra reveal that self-sustained oscillations due to shear layer-trailing edge interactions are absent or very weak. In the early development of the cavity shear layer, dominant frequencies of the velocity fluctuations do not depend on $L / D$, and the frequencies are close to those of the shear layer instability. As the measuring point moves to the downstream, oscillation frequencies decrease and the peaks of the spectra become unclear.

(3) PIV-measured instantaneous flow field show that two different structures, large- and small-scale structures, exist in the cavity shear layer. The large-scale structures consist of multiple small-scale structures. The frequencies estimated from the streamwise wavelength of the large-scale structures are close to those of the shear layer instability. However, in PIV images, streamwise spacing of the large-scale structures do not indicate the occurrences of low-frequency components observed in the velocity spectra. Further investigations, for example time-resolved PIV measurements, are recommended to clarify the mechanism of the low-frequency oscillations.

\section{References}

(1) Mizushima, F., Takakura, H., Kurita, T., Kato, C., and Iida, A., "Experimental investigation of aerodynamic noise generated by a train-car gap," J. Fluid Science and Technology, Vol.2, (2007), pp.464-479

(2) Sasajima, H., Okamura, N., and Tanida, T., "Vortex-induced vibration experienced on sea-chest," J. Society of Naval Architect of Japan, Vol.159 (1985), pp.391-404 (in Japanese)

(3) Mori, T., and Naganuma, K., "Experimental investigation of the three-dimensional nature of turbulent flow over a rectangular cavity," J. Fluid Science and Technology, Vol.4, (2009), pp.746-757

(4) Lin, J.C., and Rockwell, D., "Organized oscillations of initially turbulent flow past a cavity," AIAA J., Vol.39 (2001), pp.1139-1151

(5) Chang, K., Constantinescu, G, and Park, S., "Analysis of the flow and mass transfer process for the incompressible flow past an open cavity with a laminar and a fully turbulent incoming boundary layer," J. Fluid Mech., Vol.561 (2006), pp.113-145

(6) Kang, W., and Sung, H.J., "Experimental study of self-sustained oscillations in low Mach number turbulent cavity flows," Proceedings of $7^{\text {th }}$ international symposium on PIV, (2007)

(7) Rockwell, D., and Knisely, C., "The organized nature of flow impingement upon a corner,” J. Fluid Mech., Vol.93 (1979), pp.413-432

(8) Hyun, B.S., Balachander, R., Yu, K., and Patel, V.C., "PIV/LDV measurements of mean 
velocity and turbulence in a complex open channel flow," IIHR Technical Rep. No.424, (2003), University of IOWA

(9) Rowley, C.W., Colonius, T., and Basu, A.J., "On self-sustained oscillations in two-dimensional compressible flow over rectangular cavities," J. Fluid Mech., Vol.455 (2002), pp.315-346

(10) Brown, G.L., and Roshko, A., "On density effects and large scale structure in turbulent mixing layers," J. Fluid Mech., Vol.64 (1974), pp.775-816

(11) Adrian, R.J., Christensen, Z.C., and Liu, Z.C., "Analysis and interpretation of instantaneous turbulent velocity fields," Experiments in Fluids, 29-(2000), pp.275-290

(12) Knisely, C., and Rockwell, D., "Self sustained low-frequency components in an impinging shaer layer," J. Fluid Mech., Vol.116 (1982), pp.157-186 